\title{
Severe versus common COVID-19: an early warning nomogram model
}

\author{
Yanxin Chang ${ }^{1,2,{ }^{*}}$, Xuying Wan ${ }^{2,3,}{ }^{*}$, Xiaohui Fu ${ }^{2,4}{ }^{*}$, Ziyu Yang ${ }^{3,5}$, Zhijie Lu $^{2,6}$, Zhenmeng Wang ${ }^{2,6}$, \\ $\mathrm{Li} \mathrm{Fu}^{2,7}$, Lei Yin ${ }^{2,4}$, Yongjie Zhang ${ }^{4}$, Qian Zhang ${ }^{2,7}$ \\ ${ }^{1}$ Biliary Tract Surgery Department IV, Eastern Hepatobiliary Surgery Hospital, Second Military Medical University, \\ Shanghai 200438, PR China \\ ${ }^{2}$ Infectious Disease Department IV, Hubei Maternal and Child Health Hospital, Wuhan 430074, PR China \\ ${ }^{3}$ Department of Integrated Traditional and Western Medicine, Eastern Hepatobiliary Surgery Hospital, Second \\ Military Medical University, Shanghai 200438, PR China \\ ${ }^{4}$ Biliary Tract Surgery Department II, Eastern Hepatobiliary Surgery Hospital, Second Military Medical University, \\ Shanghai 200438, PR China \\ ${ }^{5}$ Infectious Disease Department II, Huoshenshan Hospital, Wuhan 430113, PR China \\ ${ }^{6}$ Department of Anesthesiology, Eastern Hepatobiliary Surgery Hospital, Second Military Medical University, \\ Shanghai 200438, PR China \\ ${ }^{7}$ Department of Biotherapy, Eastern Hepatobiliary Surgery Hospital, Second Military Medical University, Shanghai \\ 200438, PR China \\ *Equal contribution
}

Correspondence to: Yongjie Zhang, Qian Zhang; email: yjoy005@163.com, https://orcid.org/0000-0002-0437-9578;

zhqiank@smmu.edu.cn

Keywords: COVID-19, early warning nomogram model, severe versus common, risk factors, validation

Received: March 22, $2021 \quad$ Accepted: December 25, $2021 \quad$ Published: January 17, 2022

Copyright: (C) 2022 Chang et al. This is an open access article distributed under the terms of the Creative Commons Attribution License (CC BY 3.0), which permits unrestricted use, distribution, and reproduction in any medium, provided the original author and source are credited.

\section{ABSTRACT}

The wide spread of coronavirus disease 2019 is currently the most rigorous health threat, and the clinical outcomes of severe patients are extremely poor. In this study, we establish an early warning nomogram model related to severe versus common COVID-19. A total of 1059 COVID-19 patients were analyzed in the primary cohort and divided into common and severe according to the guidelines on the Diagnosis and Treatment of COVID-19 by the National Health Commission of China (7th version). The clinical data were collected for logistic regression analysis to assess the risk factors for severe versus common type. Furthermore, 123 COVID-19 patients were reviewed as the validation cohort to assess the performance of this model. Multivariate logistic analysis revealed that age, dyspnea, lymphocyte count, C-reactive protein and interleukin- 6 were independent factors for prewarning the severe type occurrence. Then, the early warning nomogram model including these risk factors for inferring the severe disease occurrence out of common type of COVID-19 was constructed. The C-index of this nomogram in the primary cohort was $0.863,95 \%$ confidence interval (Cl) $(0.836-0.889)$. Meanwhile, in the validation cohort, the $\mathrm{C}$-index of this nomogram was $0.889,95 \% \mathrm{Cl}(0.828-0.950)$. In both the primary cohort and validation cohorts, the calibration curve showed good agreement between prediction and actual probability. The early warning model shows that data at the very beginning including age, dyspnea, lymphocyte count, CRP, and IL-6 may prewarn the severe disease occurrence to some extent, which could help clinicians early and timely treatment. 


\section{INTRODUCTION}

Coronaviruses are single-stranded, positive-sense RNAs consisting of four genera, including alpha-coronavirus $(\alpha-\mathrm{CoV})$, beta-coronavirus $(\beta-\mathrm{CoV})$, delta-coronavirus $(\delta-\mathrm{CoV})$ and gamma-coronavirus $(\gamma-\mathrm{CoV})$, which are viruses that have caused severe global infections, such as severe acute respiratory syndrome (SARS) and Middle East respiratory syndrome (MERS) [1, 2]. As a novel beta-coronavirus, severe acute respiratory syndrome coronavirus 2 (SARS-CoV-2) is characterized by an enveloped RNA virus and pleomorphic shape with a diameter of 60-140 nm [3]. It is known as the cause of coronavirus disease 2019 (COVID-19), a highly infectious disease that has been declared a global public health emergency by the World Health Organization (WHO). The COVID-19 outbreak has rapidly transitioned into a worldwide pandemic owing to the major transmission of respiratory droplets as well as contact transmission (such as inhalation of aerosols in certain activities (e.g., singing, intubation or the use of nebulizers) or even contamination of inanimate surfaces). Up to April 26, 2020, the COVID19 pandemic caused more than 2,719,897 cases and 187,705 deaths around the world, with over 200 countries affected [4].

According to the latest version of the guidelines on the Diagnosis and Treatment of COVID-19 by the National Health Commission of China, patients infected with COVID-19 are clinically defined as mild, moderate, severe and critical [3]. Among them, mild patients present light symptoms without signs of pneumonia, and moderate patients start to show fever, respiratory symptoms and pneumonia by imaging. For severe patients, most of them develop dyspnea and/or hypoxia within one week, and even critical cases progress rapidly to acute respiratory distress symptom (ARDS), septic shock, irreversible metabolic acidosis, coagulation dysfunction and multi-organ dysfunction. Laboratory indexes show normal or reduced peripheral white blood cell (WBC) count, total lymphocyte count, increased erythrocyte sedimentation rate (ESR), and Creactive protein (CRP) and partial reduction of heparinase (Hpa), lactate dehydrogenase (LDH), creatase, and myoglobin in moderate patients. Severe patients have decreased D-dimer and progressive reduction of peripheral lymphocytes or elevated inflammatory cytokines in severe and critical patients. At present, most patients are mild and moderate at diagnosis, but a few patients can progress rapidly to severe, critical or even death [3]. Since there is still no consensus on the factors that lead to the progression from common (mild and moderate) to severe (severe and critical) disease, the clinical diagnosis and treatment of COVID-19 is difficult. Therefore, an early warning consensus of severe patient is needed to guide early and timely treatment.

Inspired by the use of predictive models for prognosis in other diseases, we retrospectively reviewed the medical records of 1059 COVID-19 patients, conducted logistic regression analysis for factors related to severe versus common type and established an early warning nomogram model including these high risk factors of severe disease occurrence in COVID-19 patients. Then, the early warning nomogram model was validated in another cohort including 123 COVID-19 patients.

\section{RESULTS}

\section{COVID-19 patient characteristics}

In the primary cohort, a total of 1059 COVID-19 patients were enrolled, and there were 808 common patients (including $326(40.3 \%)$ males and 482 (59.7\%) females) and 251 severe patients (including 133 $(53.0 \%)$ males and $118(47.0 \%)$ females). In the validation cohort, a total of 123 COVID-19 patients were recruited, including 90 common patients (including 46 (51.1\%) males and 44 (48.9\%) females) and 33 severe patients (including $21(63.6 \%)$ males and $12(36.4 \%)$ females). The clinical characteristics and laboratory findings of COVID-19 patients in the primary and validation cohorts are listed in Table 1.

\section{Risk factors of the severe type of COVID-19}

Univariate logistic regression analysis showed that age ( $\geq 60$ years $V<60$ years) $(P<0.001)$, dyspnea (yes $V$ no) $(P<0.001)$, lymphocyte count (low $V$ normal) $(P=$ $0.001)$, CRP (high $V$ normal) $(P<0.001)$ and IL-6 (high $V$ normal $)(P<0.001)$ were correlated with the occurrence of severe type out of the common type of COVID-19 (Table 2).

\section{Independent factors for prewarning the severe type of COVID-19}

Multivariate logistic regression analysis disclosed that age ( $\geq 60$ years $V<60$ years) $(P<0.001)$, dyspnea (yes $V$ no) $(P<0.001)$, lymphocyte count (low $V$ normal) $(P$ $<0.001)$, CRP (high $V$ normal) $(P<0.001)$ and IL-6 (high $V$ normal) $(P<0.001)$ were independent factors in prewarning the severe disease occurrence of COVID-19 (Table 3), which may help clinicians early and timely treatment.

\section{Early warning nomogram construction for severe type occurrence}

The early warning nomogram was constructed based on the independent factors for inferring the severe disease 
Table 1. Clinical characteristics and laboratory findings of patients with COVID-19 in primary cohort and validation cohort.

\begin{tabular}{|c|c|c|c|c|}
\hline \multirow{3}{*}{ Features } & \multicolumn{2}{|c|}{ Primary cohort $(N=1059)$} & \multicolumn{2}{|c|}{ Validation cohort $(N=123)$} \\
\hline & Common type $(n=808)$ & Severe type $(n=251)$ & Common type $(n=90)$ & Severe type $(n=33)$ \\
\hline & No. $(\%)$ & No. $(\%)$ & No. $(\%)$ & No. $(\%)$ \\
\hline \multicolumn{5}{|l|}{ Sex } \\
\hline Male & $326(40.3)$ & $133(53.0)$ & $46(51.1)$ & $21(63.6)$ \\
\hline Female & $482(59.7)$ & $118(47.0)$ & $44(48.9)$ & $12(36.4)$ \\
\hline \multicolumn{5}{|l|}{ Age, years } \\
\hline$<60$ & $439(54.3)$ & $58(23.1)$ & $66(73.3)$ & $6(18.2)$ \\
\hline$\geq 60$ & $369(45.7)$ & $193(76.9)$ & $24(26.7)$ & $27(81.8)$ \\
\hline \multicolumn{5}{|l|}{ Weight, kg } \\
\hline$<64$ & $409(50.6)$ & $165(65.7)$ & $46(51.1)$ & $15(45.5)$ \\
\hline$\geq 64$ & $399(49.4)$ & $86(34.3)$ & $44(48.9)$ & $18(54.5)$ \\
\hline \multicolumn{5}{|l|}{ Symptoms } \\
\hline Dyspnea & $191(23.6)$ & $140(55.8)$ & $17(18.9)$ & $21(63.6)$ \\
\hline Fever & $535(66.2)$ & $166(66.1)$ & $54(60.0)$ & $21(63.6)$ \\
\hline Cough & $424(52.5)$ & $127(50.6)$ & $58(64.4)$ & $18(54.5)$ \\
\hline Fatigue & $215(26.6)$ & $72(28.7)$ & $32(35.6)$ & $9(27.3)$ \\
\hline \multicolumn{5}{|c|}{ Laboratory tests } \\
\hline \multicolumn{5}{|c|}{ Leukocyte count, $\times 10 / \mathrm{L}$} \\
\hline$<9.5$ & $781(96.7)$ & $217(86.5)$ & $88(97.8)$ & $32(97.0)$ \\
\hline$\geq 9.5$ & $27(3.3)$ & $34(13.5)$ & $2(2.2)$ & $1(3.0)$ \\
\hline \multicolumn{5}{|c|}{ Lymphocyte count, $\times 10 / \mathrm{L}$} \\
\hline$<1.1$ & $110(13.6)$ & $115(45.8)$ & $12(13.3)$ & $14(42.4)$ \\
\hline$\geq 1.1$ & $698(86.4)$ & $136(54.2)$ & $78(86.7)$ & $19(57.6)$ \\
\hline \multicolumn{5}{|c|}{ Neutrophil count, $\times 10 / \mathrm{L}$} \\
\hline$<6.3$ & $776(96.0)$ & $200(79.7)$ & $88(97.8)$ & $28(84.8)$ \\
\hline$\geq 6.3$ & $32(4.0)$ & $51(20.3)$ & $2(2.2)$ & $5(15.2)$ \\
\hline \multicolumn{5}{|c|}{ Monocytes count, $\times 10 / \mathrm{L}$} \\
\hline$<0.6$ & 759 (93.9) & $230(91.6)$ & $85(94.4)$ & $32(97.0)$ \\
\hline$\geq 0.6$ & $49(6.1)$ & $21(8.4)$ & $5(5.6)$ & $1(3.0)$ \\
\hline \multicolumn{5}{|l|}{$\mathrm{CRP}, \mathrm{mg} / \mathrm{L}$} \\
\hline$<0.6$ & $668(82.7)$ & $94(37.5)$ & $84(93.3)$ & $18(54.5)$ \\
\hline$\geq 0.6$ & $140(17.3)$ & $157(62.5)$ & $6(6.7)$ & $15(45.5)$ \\
\hline \multicolumn{5}{|l|}{$\mathrm{ALT}, \mathrm{U} / \mathrm{L}$} \\
\hline$<55$ & $735(91.0)$ & $207(82.5)$ & $85(94.4)$ & $28(84.8)$ \\
\hline$\geq 55$ & $73(9.0)$ & $44(17.5)$ & $5(5.6)$ & $5(15.2)$ \\
\hline \multicolumn{5}{|l|}{ AST, U/L } \\
\hline$<34$ & $752(93.1)$ & $208(82.9)$ & $86(95.6)$ & $24(72.7)$ \\
\hline$\geq 34$ & $56(6.9)$ & $43(17.1)$ & $4(4.4)$ & $9(27.3)$ \\
\hline \multicolumn{5}{|l|}{$\mathrm{ALP}, \mathrm{U} / \mathrm{L}$} \\
\hline$<79$ & $600(74.3)$ & $147(58.6)$ & $73(81.1)$ & $20(60.6)$ \\
\hline$\geq 79$ & $208(25.7)$ & $104(41.4)$ & $17(18.9)$ & $13(39.4)$ \\
\hline \multicolumn{5}{|c|}{ TBIL, $\mu \mathrm{mol} / \mathrm{L}$} \\
\hline$<20.5$ & $769(95.2)$ & $227(90.4)$ & $87(96.7)$ & $30(90.9)$ \\
\hline$\geq 20.5$ & $39(4.8)$ & $24(9.6)$ & $3(3.3)$ & $3(9.1)$ \\
\hline PCT, ng/ml & & & & \\
\hline
\end{tabular}




\begin{tabular}{lcccc}
$<0.04$ & $647(80.1)$ & $140(55.8)$ & $87(96.7)$ & $30(90.9)$ \\
$\geq 0.04$ & $161(19.9)$ & $111(44.2)$ & $3(3.3)$ & $3(9.1)$ \\
$\begin{array}{l}\text { IL-6, } \mathrm{pg} / \mathrm{mL} \\
<10\end{array}$ & $781(96.7)$ & $169(67.3)$ & $87(96.7)$ & $20(60.6)$ \\
$\geq 10$ & $27(3.3)$ & $82(32.7)$ & $3(3.3)$ & $13(39.4)$ \\
\hline
\end{tabular}

Abbreviations: COVID-19: Corona Virus Disease 2019; CRP: C-reactive protein; ALT: alanine aminotransferase; AST: aspartate aminotransferase; ALP: Alkaline phosphatase; TBIL: total bilirubin; PCT: procalcitonin; IL-6: interleukin 6.

Table 2. Univariate logistic regression analysis of factors related to the progression from common type to severe type of COVID-19.

\begin{tabular}{lccccc}
\hline \multirow{2}{*}{ Variables } & \multicolumn{5}{c}{ Univariate logistic regression model } \\
\cline { 2 - 5 } & B & S.E. & P value & OR & 95\% CI \\
\hline Sex (male $V$ female) & 0.218 & 0.197 & 0.268 & 1.243 & $0.846-1.828$ \\
Age $(\geq 60$ years $V<60$ years) & 1.173 & 0.198 & $<0.001$ & 3.233 & $2.193-4.765$ \\
Weight $(\geq 64$ kg $V<64 \mathrm{~kg}$ ) & 0.215 & 0.201 & 0.285 & 1.239 & $0.837-1.836$ \\
Dyspnea (yes $V$ no) & 1.325 & 0.189 & $<0.001$ & 3.761 & $2.599-5.443$ \\
Fever (yes $V$ no) & -0.180 & 0.195 & 0.355 & 0.835 & $0.570-1.223$ \\
Cough (yes $V$ no) & -0.104 & 0.183 & 0.572 & 0.901 & $0.629-1.292$ \\
Fatigue (yes $V$ no) & -0.123 & 0.210 & 0.559 & 0.884 & $0.586-1.335$ \\
Leukocyte count (high $V$ normal) & -0.291 & 0.545 & 0.593 & 0.747 & $0.257-2.175$ \\
Lymphocyte count (low $V$ normal) & 0.719 & 0.212 & 0.001 & 2.052 & $1.355-3.108$ \\
Neutrophil count (high $V$ normal) & 0.790 & 0.480 & 0.100 & 2.203 & $0.859-5.645$ \\
Monocytes count (high $V$ normal) & -0.577 & 0.363 & 0.112 & 0.562 & $0.275-1.145$ \\
CRP (high $V$ normal) & 1.242 & 0.215 & $<0.001$ & 3.464 & $2.274-5.274$ \\
ALT (high $V$ normal) & 0.514 & 0.335 & 0.124 & 1.673 & $0.868-3.223$ \\
AST (high $V$ normal) & -0.303 & 0.362 & 0.402 & 0.738 & $0.363-1.502$ \\
ALP (high $V$ normal) & 0.050 & 0.204 & 0.806 & 1.051 & $0.705-1.567$ \\
TBIL (high $V$ normal) & -0.049 & 0.385 & 0.898 & 0.952 & $0.447-2.025$ \\
PCT (high $V$ normal) & 0.345 & 0.205 & 0.092 & 1.412 & $0.945-2.111$ \\
IL-6 (high $V$ normal) & 1.376 & 0.303 & $<0.001$ & 3.960 & $2.186-7.171$ \\
\hline
\end{tabular}

Abbreviations: COVID-19: Corona Virus Disease 2019; B: regression coefficient; S.E.: standard error; OR: odds ratio; Cl: confidence interval; CRP: C-reactive protein; ALT: alanine aminotransferase; AST: aspartate aminotransferase; ALP: Alkaline phosphatase; TBIL: total bilirubin; PCT: procalcitonin; IL-6: interleukin 6.

occurrence out of common type of COVID-19. As shown in Figure 1, each variable could be assessed vertically to obtain the point, and the total points of all five variables were calibrated correspondingly to the risk. Age was the strongest contributor to the progression from common type to severe type COVID19 , followed by dyspnea, CRP, IL-6 and lymphocyte count. The C-index of this nomogram was $0.863,95 \%$ CI (0.836-0.889). In addition, the early warning accuracy of this nomogram in the primary cohort is illustrated in Figure 2A, the calibration curve showed excellent agreement between prediction and actual probability. Also, the receiver operating characteristic
(ROC) curve was employed to assess the predictive ability of the established nomogram, and the area under curve (AUC) was 0.863 in the training cohort, with a sensitivity of $72.1 \%$ and specificity of $86.4 \%(P<0.001$, Figure 3A). Validation of the early warning accuracy of the
nomogram for disease progression

The accuracy of the nomogram for disease progression was validated in another cohort including 123 patients. In the calibration curve of Figure 2B, the C-index of this nomogram in the validation cohort was 0.889 , 
Table 3. Multivariate logistic regression analysis of factors related to the progression from common type to severe type of COVID-19.

\begin{tabular}{lccccc}
\hline \multirow{2}{*}{ Variables } & \multicolumn{5}{c}{ Multivariate logistic regression model } \\
\cline { 2 - 5 } & B & S.E. & P value & OR & 95\% CI \\
\hline Age $(\geq 60$ years $V<60$ years) & 1.124 & 0.193 & $<0.001$ & 3.078 & $2.107-4.496$ \\
Dyspnea (yes $V$ no) & 1.336 & 0.181 & $<0.001$ & 3.802 & $2.665-5.425$ \\
Lymphocyte count (low $V$ normal) & 0.842 & 0.202 & $<0.001$ & 2.320 & $1.561-3.449$ \\
CRP (high $V$ normal) & 1.374 & 0.199 & $<0.001$ & 3.953 & $2.676-5.839$ \\
IL-6 (high $V$ normal) & 1.451 & 0.285 & $<0.001$ & 4.267 & $2.443-7.453$ \\
\hline
\end{tabular}

Abbreviations: COVID-19: Corona Virus Disease 2019; B: regression coefficient; S.E.: standard error; OR: odds ratio; Cl: confidence interval; CRP: C-reactive protein; IL-6: interleukin 6.

95\% CI (0.828-0.950). The calibration curve showed good agreement between the prediction and actual probability. Moreover, in the validation cohort, the AUC of ROC curve was 0.889 with a sensitivity of $93.9 \%$ and specificity of $87.8 \%(P<0.001$, Figure $3 \mathrm{~B})$. In both the primary cohort and validation cohort, the calibration plots presented good consistency between prediction and actual probability. Thus, the nomogram showed good accuracy and discrimination ability in early identifying the occurrence of severe disease out of common type of COVID-19.

\section{Clinical application of the early warning nomogram}

In order to assess the clinical applicability of the risk prediction nomogram, Decision curve analysis (DCA) based on the net benefit and threshold probabilities was performed. As shown in Figure 4, whether in primary or

Points

$$
\begin{array}{lllllllllll}
0 & 10 & 20 & 30 & 40 & 50 & 60 & 70 & 80 & 90 & 100
\end{array}
$$

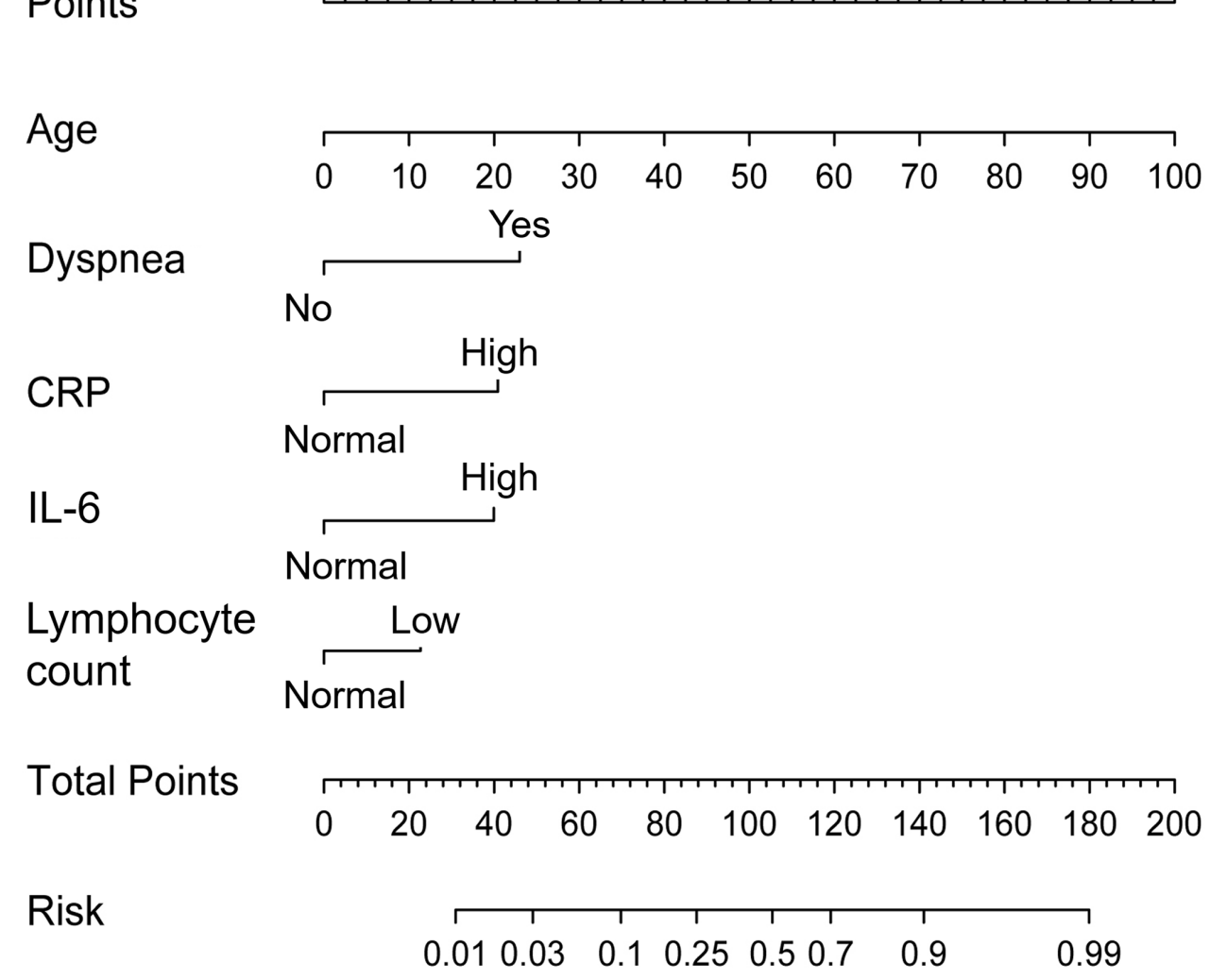

Figure 1. Risk prewarning nomogram for severe type patients. Each patient's variables could be located on the corresponding variable axis. The point of each variable could be determined by vertically referring to the top point line. By summing up the total points of each corresponding variables, total point was calculated, and risk of disease progression was determined by reading against the risk axis. 
validation cohort, our risk prediction nomogram had a superior net benefit with a wide range of threshold probabilities.

\section{DISCUSSION}

There is a large difference in prognosis between common patients and severe patients. A study on

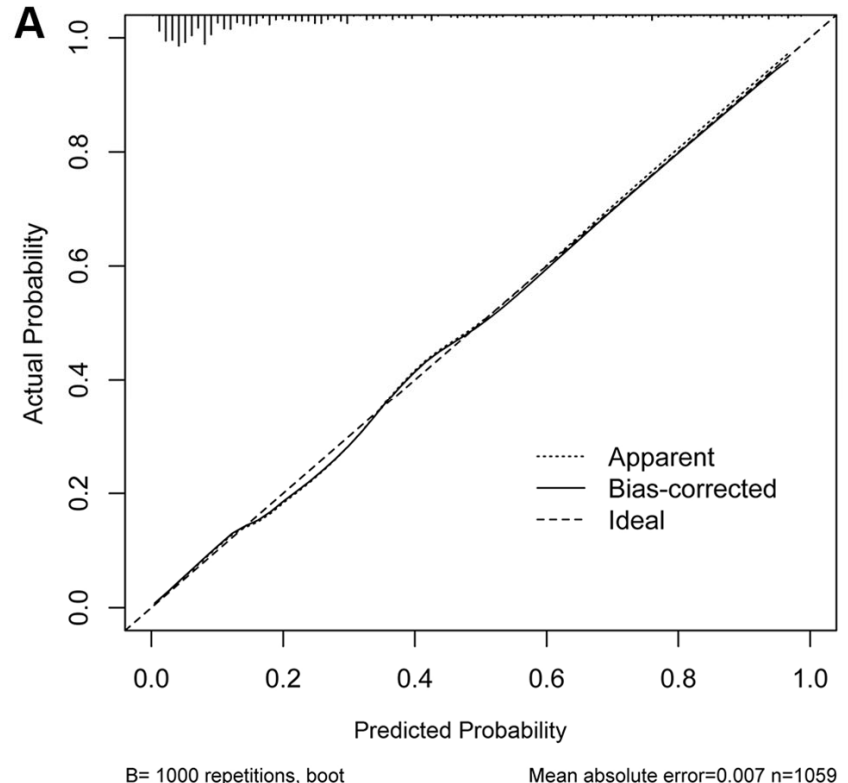

COVID-19 patients from Jinyintan Hospital and Wuhan Pulmonary Hospital reported that severe and critical patients show significantly higher mortality than general patients [5]. The in-hospital observations also show high mortality in patients admitted to extracorporeal membrane oxygenation (ECMO) and a rapid drop in $\mathrm{SaO}$ in severe patients. Therefore, investigating the influential factors that underlie the transition of

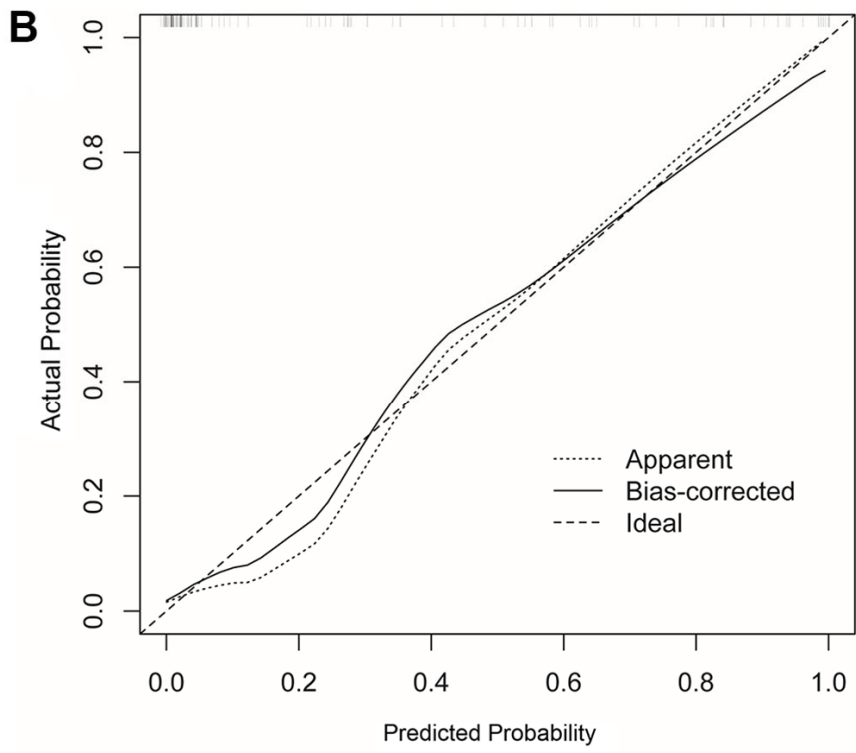

$B=1000$ repetitions, boot

Mean absolute error $=0.019 n=123$

Figure 2. The calibration curves of nomogram in prewarning the severe infection occurrence. Nomogram predicted severe type risk was plotted on $\mathrm{x}$-axis, the actual disease progression probability was plotted on y axis. (A) Training cohort; (B) Validation cohort.
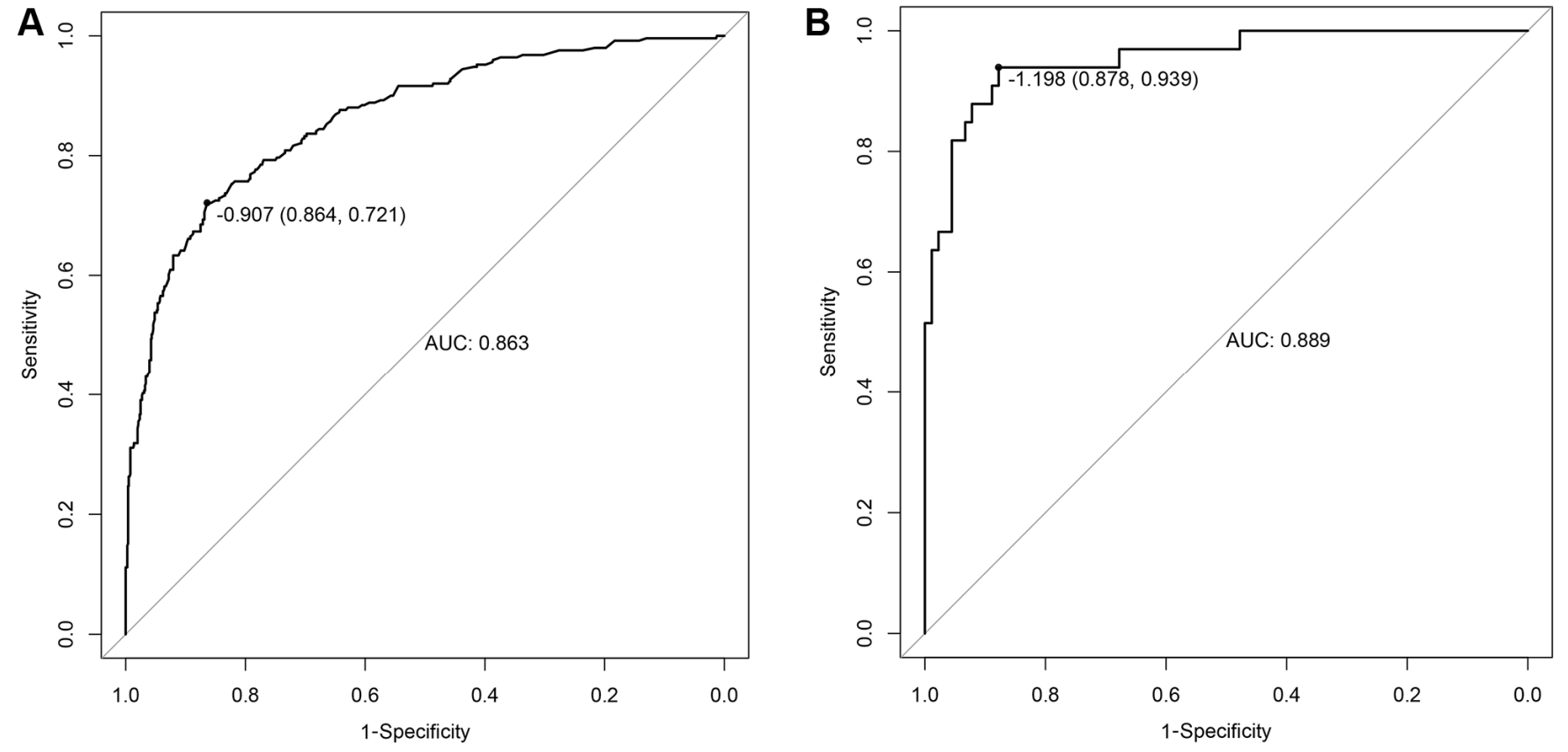

Figure 3. The ROC curves of the nomogram. (A) Training cohort; (B) Validation cohort. 
common to severe disease is extremely important for the early identification of patients at risk as well as time management. We believe that the progression of common to severe disease occurs earlier physiologically. For instance, some patients had sharp deterioration soon after slightly increased physical activity. This indicated that the disease had already progressed over the common threshold to severe before the patient started to show severe symptoms. As analyzed by existing evidence, there are risk factors for mortality in COVID-19 patients, including older age, high D-dimer level and high Sequential Organ Failure Assessment (SOFA) score at admission [5]. In addition, the signs for ICU admission, including dyspnea, low WBC count, high neutrophil count and D-dimer level, are also critical references for the diagnosis of severe disease [6]. According to these risk factors for ICU admission as well as mortality, it is presumable that analyzing patients' characteristics at hospital admission could help with identifying common to severe progression and may aid appropriate supportive care and prompt access to the ICU if necessary. Considering the clinical feasibility, we comprehensively extracted key clinical features of 1059 COVID patients, including sex, age, weight, symptoms and laboratory tests, conducted logistic regression analysis and successfully constructed an early warning model for severe disease occurrence.

Herein, we discovered that age $(\geq 60$ years $V<60$ years), dyspnea (yes $V$ no), lymphocyte count (low $V$

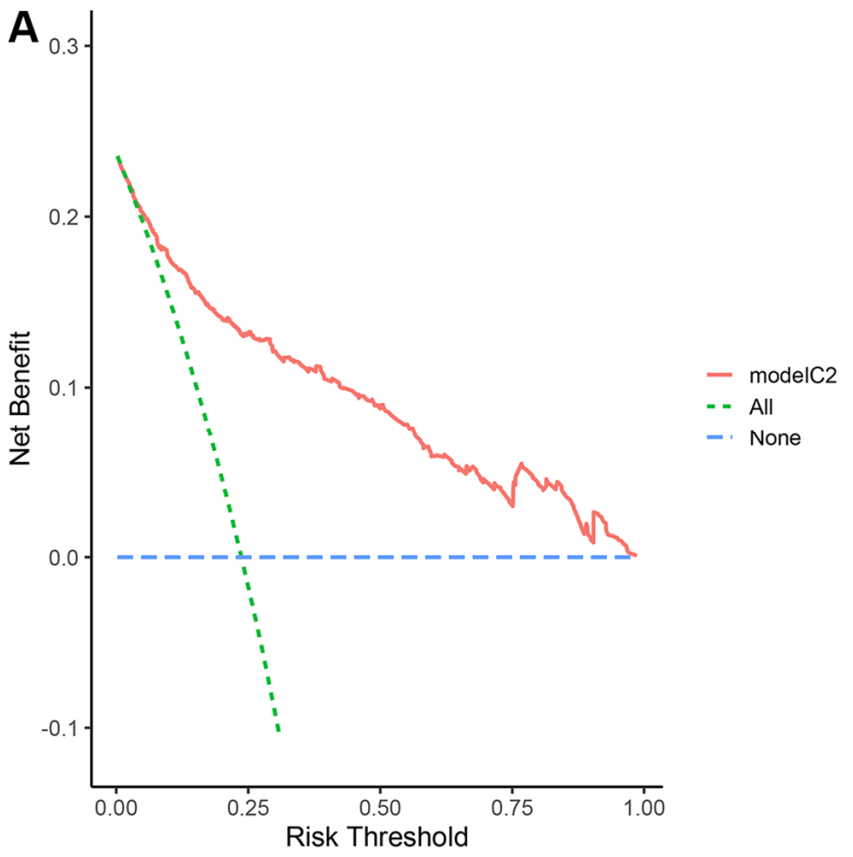

normal), CRP (high $V$ normal) and IL-6 (high $V$ normal) were independent factors to infer the severe disease occurrence out of common type of COVID-19, in which age was the most important contributor. In terms of age ( $\geq 60$ years $V<60$ years), older patients featured a poor physical state and decreased immune function, which made them vulnerable to infections and difficult to quickly relieve in the face of COVID-19, subsequently prolonging the disease course to progress rapidly to severe or critical or even death $[7,8]$. In addition, older patients usually have several comorbidities (including hypertension [9], diabetes [10] and cardiovascular disease [11]. Considering that lung infections caused by this novel coronavirus might increase the burden on the heart and cause high blood sugar, the characteristics of multisystem disease coexisting in the elderly lead to complicated and complex diseases. Multiple diseases affect each other, and the difficulty of treatment is greatly increased. Thus, elderly patients are prone to multisystem organ dysfunction and even failure, thereby progressing rapidly to severe or critical or even death [7]. Older age could mostly infer the severe disease occurrence out of common type of COVID- 19 .

Regarding dyspnea (yes $V$ no), SARS-CoV-2 entered the lungs via respiration and then activated immune cells, cytokines and other pathogen-resistance systems [12]. With an increase in virus virulence, patient immunity might decrease during the struggle between the virus and the human host, which might result in congestion and edema in lung tissue, thickening of the

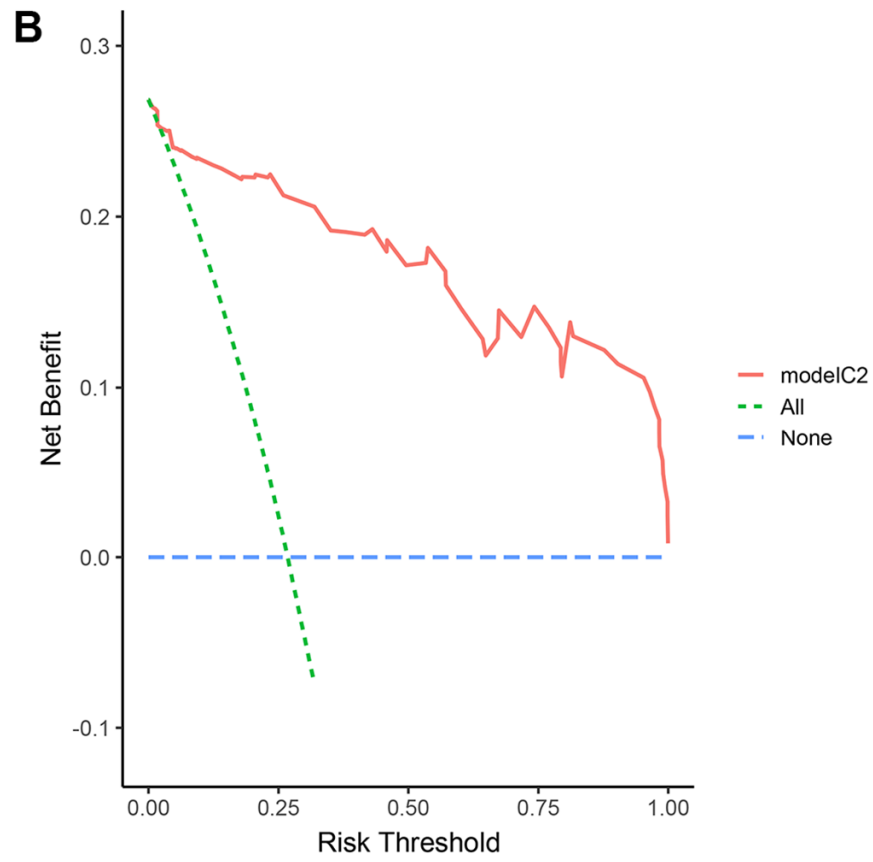

Figure 4. DCA curves of the nomogram. DCA compares the net benefits of three scenarios in prewarning the severe disease occurrence: A perfect prediction model (blue line), screen none (horizontal green line), and screen based on the nomogram (red line). The DCA curves were depicted in the training cohort (A), validation cohort (B). 
interstitial lung and increased exudation in the alveolar space to form a transparent membrane-like structure [12]. These severe pathological changes in lung tissue might directly make patients feel dyspnea. Hence, dyspnea could be an independent factor to prewarn the severe disease occurrence out of common type of COVID-19.

In terms of lymphocyte count (low $V$ normal), after SARS-CoV-2 infection, lymphopenia is a typical laboratory abnormality, which is similar to other highly pathogenic coronavirus infections (including SARSCoV [13] and MERS-CoV [14] infections). Lymphocyte subsets play an important role in cellular immune regulation, and SARS-CoV-2 infection has been reported to primarily impact $\mathrm{T}$ lymphocytes (especially CD4+ $\mathrm{T}$ and $\mathrm{CD} 8+\mathrm{T}$ cells), which means that $\mathrm{T}$ lymphocytes might be highly involved in the pathological process of COVID-19 and provide an important defense against COVID-19 [12, 15]. Additionally, its magnitude of decrease suggested the extent of impairment of the immune system by viral infection $[12,16]$. Thus, lymphocyte count might act as a powerful factor for independently indicating the severe disease occurrence out of common type of COVID-19.

CRP and IL-6, two common proinflammatory factors, have been confirmed to be associated with severe lung injury and adverse clinical outcomes of SARS-CoV or MERS-CoV infection [14, 17-19]. After SARS-CoV-2 infection, CRP and IL-6 also increased rapidly in a short time [12], which is in line with the concept of a "cytokine storm," and the magnitude of the cytokine storm is associated with disease severity [16]. Therefore, increased CRP and IL-6 might be related to the disease severity of COVID-19. In addition, CRP and IL-6 might directly accelerate protein degradation and indirectly influence important metabolic pathways to make patients frail [20], thereby causing patients to progress rapidly to severe or critical infection or even death. CRP and IL-6 might serve as independent factors prewarning the severe infection occurrence. However, considering that the understanding of COVID-19 is still insufficient and the detailed reasons for the role of these factors in COVID-19 patients is unclear, further studies are needed.

The widespread use of COVID-19 in the global population is currently the most rigorous health threat. Although most cases are mild, the apparently large number of infections poses a considerable burden to the national medical resources in affected countries. In addition, since the window of common to severe transition is short and the clinical outcomes of severe patients are extremely poor, an increasing number of lives have been lost due to delay of life support and treatment. Early warning biomarkers are urgently needed to assist with timely treatment and early prevention of disease aggravation. The nomogram model showed good agreement between prediction and actual probability in the primary cohort as well as the validation cohort. Patients who are high risk based on the nomogram model could be closely monitored for clinical index during disease course, and early intervention could be initiated to shorten the time interval between the onset of severe disease and treatment, which increases the survival possibility.

There are some limitations of our study. First, the sample size for nomogram validation was relatively small. More cases as well as a prospectively designed study are needed to further prove the accuracy of our nomogram model. Second, the phylogenetic analysis revealed three different variants of SARS-CoV-2 that might present distinct disease severity, whereas the virus variant was not differentiated in this study, which might influence the accuracy of the early warning model [21]. Third, since different centers were involved and the treatment concept in different centers might differ due to the relatively urgent medical situation, the clinical outcomes might vary. Furthermore, a comprehensive and in-depth understanding of COVID19 is still lacking regarding the pathology and virus source; therefore, the current findings need supportive data from broader and global analyses.

In conclusion, the nomogram (including age, dyspnea, lymphocyte count, CRP and IL-6) objectively and accurately prewarns the severe disease occurrence out of common type COVID-19 patients. Our findings provide good evidence to accurately estimate changes in the patient's condition, thereby aiding prevention and advance treatment.

\section{METHODS}

\section{Study design and patients}

Between February 19, 2020 and April 7, 2020, 1059 COVID-19 patients admitted to Guanggu District, Hubei Maternal and Child Health Hospital were analyzed in this study. All the clinical characteristics were collected in the incipient stage when all the patients were diagnosed as common type COVID-19. During the period from hospitalization to discharge, part of patients changed into severe type. All patients with laboratory-confirmed SARS-CoV-2 infection were diagnosed with COVID-19 according to the diagnostic criteria proposed in the 7th version of the Guidelines on the Diagnosis and Treatment of COVID-19 by the National Health Commission of China (available at: http://www.nhc.gov.cn/). The laboratory-confirmed 
SARS-CoV-2 infection was defined as a positive result of a real-time reverse transcriptase polymerase chain reaction (RT-PCR) assay of nasal-pharyngeal swab sample or/and a positive result of SARS-CoV-2 specific IgM antibody and IgG antibody in a serum sample. All 1059 COVID-19 patients formed the primary cohort, and all of them had complete clinical data, including basic characteristics and laboratory tests, which were used as a training dataset for constructing the disease progression early warning model, fitting the parameters and internal validation. Furthermore, a total of 123 COVID-19 patients admitted to Huoshenshan (Fire God Mountain) Hospital were enrolled in the study. All 123 patients also had a confirmed diagnosis of COVID-19, similar to the patients in the primary cohort, and served as the validation cohort in the study. The clinical data of these 123 patients were used as a test dataset for external validation to assess the performance of this early warning model.

\section{Clinical classification for COVID-19}

Clinical classification for COVID-19 was performed according to the 7th version of the Guidelines on the Diagnosis and Treatment of COVID-19 by the National Health Commission of China, which were as follows: (1) Mild type: the clinical symptoms were mild with no abnormal radiological findings; (2) moderate type: fever and respiratory symptoms were presented with pneumonia on chest computed tomography; (3) severe type: one of the following conditions had to be met: (a) respiratory distress, respiratory rate $\geq 30$ per $\mathrm{min}$; (b) oxygen saturation on quiescent condition $\leq 93 \%$; (c) partial pressure of oxygen in arterial blood/fraction of inspired oxygen $\left(\mathrm{PaO}_{2} / \mathrm{FiO}_{2}\right) \leq 300 \mathrm{mmHg}(1 \mathrm{mmHg}=$ $0.133 \mathrm{kPa}$ ); and (4) critical type: one of the following conditions had to be met: (a) respiratory failure occurred and mechanical ventilation was required; (b) shock occurred; (c) patients with other organ dysfunction needing intensive care unit (ICU) monitoring and treatment. In the present study, there were 808 common type patients and 251 severe type patients in the primary cohort; there were 90 common type patients and 33 severe type patients in the validation cohort.

\section{Data collection}

Clinical data of patients were collected from the database of hospitals, which included the following: sex, age, weight, symptoms (dyspnea, fever, cough, fatigue), laboratory tests (leukocyte count, lymphocyte count, neutrophil count, monocyte count, c-reactive protein (CRP), alanine aminotransferase (ALT), aspartate aminotransferase (AST), alkaline phosphatase (ALP), total bilirubin (TBIL), procalcitonin (PCT), and interleukin 6 (IL-6).

\section{Statistical analysis}

SPSS 22.0 statistical software (IBM, Chicago, Illinois, USA) was used to perform the descriptive analysis and the univariate/multivariate logistic regression analysis. In the descriptive analysis, continuous variables were switched to dichotomous variables, among which age and weight were processed as dichotomous variables by the median value, and the laboratory indexes were processed as dichotomous variables by the normal range of each index. In the logistic regression analysis, only variables with a $P$ value $<0.05$ in the univariate logistic regression analysis were further included in the multivariate logistic regression analysis with the Stepwise method to construct the severe disease early warning model. The rms package in R X64 3.6.3 (http://cran.rstudio.com/bin/windows/base/) was used to formulate the nomogram based on the multivariate logistic regression model (Supplementary Material) [22]. The predictive accuracy and discriminative ability of the nomogram were determined by the concordance index (C-index) and calibration curve. The performance and accuracy of the established nomogram were assessed by receiver operating characteristic (ROC) curve by $p R O C$ package in $\mathrm{R}$ (Supplementary Material). The area under ROC (AUC) were determined. Decision curve analysis (DCA) based on the net benefit was depicted by the package of $g g D C A$ in $\mathrm{R}$ (Supplementary Material). A $P$ value $<0.05$ was considered statistically significant.

\section{AUTHOR CONTRIBUTIONS}

Y.C., X.W. and X.F. are responsible for the study design, interpretation of data, and integrity and accuracy of the data. Z.Y., Z.L., Z.W., L.F., and L.Y. are responsible for data collection and analysis. Y.C., Y.Z. and Q.Z. are responsible for writing the report.

\section{ACKNOWLEDGMENTS}

We greatly appreciate Guanggu District, Hubei Maternal and Child Health Hospital, Huoshenshan (Fire God Mountain) Hospital for support of patient data. We acknowledge all our colleagues who helped us during the current study and health care workers participating in the diagnosis and treatment of patients in Wuhan. We thank Jiangsu Hengrui Medicine Co., Ltd., Jiangsu Province, China, and Shanghai Qeejen Biotechnology Co., Ltd., Shanghai, China for assisting with data analysis.

\section{CONFLICTS OF INTEREST}

The authors declare no conflicts of interest related to this study. 


\section{FUNDING}

This study is funded by the National Natural Science Foundation of China $(81572869,81402428)$, Shanghai Health and Health Commission Project (201940492), Talent training program for young doctors of Meng Chao talent plan and Science and Technology Commission of Shanghai Municipality (20ZR1470400).

\section{REFERENCES}

1. Abd El-Aziz TM, Stockand JD. Recent progress and challenges in drug development against COVID-19 coronavirus (SARS-CoV-2) - an update on the status. Infect Genet Evol. 2020; 83:104327. https://doi.org/10.1016/i.meegid.2020.104327 PMID: $\underline{32320825}$

2. Fan C, Lei D, Fang C, Li C, Wang M, Liu Y, Bao Y, Sun Y, Huang J, Guo Y, Yu Y, Wang S. Perinatal Transmission of 2019 Coronavirus Disease-Associated Severe Acute Respiratory Syndrome Coronavirus 2: Should We Worry? Clin Infect Dis. 2021; 72:862-4. https://doi.org/10.1093/cid/ciaa226 PMID: $\underline{32182347}$

3. Diagnosis and Treatment Protocol for Novel Coronavirus Pneumonia. (Trial Version 7). Chin Med J (Engl). 2020; 133:1087-95. https://doi.org/10.1097/CM9.0000000000000819 PMID: $\underline{32358325}$

4. WHO. Coronavirus disease 2019 (COVID-19) Situation Report-94. 2020.

https://www.who.int/emergencies/diseases/novelcoronavirus-2019/situation-reports.

5. Zhou F, Yu T, Du R, Fan G, Liu Y, Liu Z, Xiang J, Wang Y, Song B, Gu X, Guan L, Wei Y, Li H, et al. Clinical course and risk factors for mortality of adult inpatients with COVID-19 in Wuhan, China: a retrospective cohort study. Lancet. 2020; 395:1054-62.

https://doi.org/10.1016/S0140-6736(20)30566-3 PMID: $\underline{32171076}$

6. Huang $C$, Wang $Y$, Li X, Ren L, Zhao J, Hu Y, Zhang L, Fan G, Xu J, Gu X, Cheng Z, Yu T, Xia J, et al. Clinical features of patients infected with 2019 novel coronavirus in Wuhan, China. Lancet. 2020; 395:497-506. https://doi.org/10.1016/S0140-6736(20)30183-5 PMID:31986264

7. Liu K, Chen $Y$, Lin R, Han K. Clinical features of COVID19 in elderly patients: A comparison with young and middle-aged patients. J Infect. 2020; 80:e14-8. https://doi.org/10.1016/j.jinf.2020.03.005 PMID:32171866

8. Clegg A, Young J, lliffe S, Rikkert MO, Rockwood K. Frailty in elderly people. Lancet. 2013; 381:752-62.
https://doi.org/10.1016/S0140-6736(12)62167-9 PMID:23395245

9. Lee JH, Kim KI, Cho MC. Current status and therapeutic considerations of hypertension in the elderly. Korean J Intern Med. 2019; 34:687-95. PMID:31272140

10. Mordarska K, Godziejewska-Zawada M. Diabetes in the elderly. Prz Menopauzalny. 2017; 16:38-43. https://doi.org/10.5114/pm.2017.68589 PMID:28721127

11. Yazdanyar A, Newman AB. The burden of cardiovascular disease in the elderly: morbidity, mortality, and costs. Clin Geriatr Med. 2009; 25:563-77.

https://doi.org/10.1016/i.cger.2009.07.007 PMID:19944261

12. Wan $S$, Yi Q, Fan $S$, Lv J, Zhang $X$, Guo L, Lang $C$, Xiao Q, Xiao K, Yi Z, Qiang M, Xiang J, Zhang B, et al. Relationships among lymphocyte subsets, cytokines, and the pulmonary inflammation index in coronavirus (COVID-19) infected patients. Br J Haematol. 2020; 189:428-37.

https://doi.org/10.1111/bjh.16659

PMID: 32297671

13. de Wit E, van Doremalen N, Falzarano D, Munster VJ. SARS and MERS: recent insights into emerging coronaviruses. Nat Rev Microbiol. 2016; 14:523-34. https://doi.org/10.1038/nrmicro.2016.81 PMID:27344959

14. Chien JY, Hsueh PR, Cheng WC, Yu CJ, Yang PC. Temporal changes in cytokine/chemokine profiles and pulmonary involvement in severe acute respiratory syndrome. Respirology. 2006; 11:715-22. https://doi.org/10.1111/j.1440-1843.2006.00942.x PMID:17052299

15. Chen G, Wu D, Guo W, Cao $Y$, Huang D, Wang $H$, Wang $T$, Zhang $X$, Chen $H, Y u H$, Zhang $X$, Zhang $M$, Wu $S$, et al. Clinical and immunological features of severe and moderate coronavirus disease 2019. J Clin Invest. 2020; 130:2620-9. https://doi.org/10.1172/JCl137244 PMID: 32217835

16. Liu J, Li S, Liu J, Liang B, Wang X, Wang H, Li W, Tong Q, Yi J, Zhao L, Xiong L, Guo C, Tian J, et al. Longitudinal characteristics of lymphocyte responses and cytokine profiles in the peripheral blood of SARSCoV-2 infected patients. EBioMedicine. 2020; 55:102763.

https://doi.org/10.1016/i.ebiom.2020.102763 PMID: 32361250

17. Chu $\mathrm{H}$, Zhou J, Wong BH, Li C, Cheng ZS, Lin X, Poon VK, Sun T, Lau CC, Chan JF, To KK, Chan KH, Lu L, et al. 
Productive replication of Middle East respiratory syndrome coronavirus in monocyte-derived dendritic cells modulates innate immune response. Virology. 2014; 454-455:197-205.

https://doi.org/10.1016/j.virol.2014.02.018

PMID:24725946

18. Zhou J, Chu H, Li C, Wong BH, Cheng ZS, Poon VK, Sun T, Lau CC, Wong KK, Chan JY, Chan JF, To KK, Chan KH, et al. Active replication of Middle East respiratory syndrome coronavirus and aberrant induction of inflammatory cytokines and chemokines in human macrophages: implications for pathogenesis. J Infect Dis. 2014; 209:1331-42.

https://doi.org/10.1093/infdis/jit504

PMID:24065148

19. Kong SL, Chui P, Lim B, Salto-Tellez M. Elucidating the molecular physiopathology of acute respiratory distress syndrome in severe acute respiratory syndrome patients. Virus Res. 2009; 145:260-9. https://doi.org/10.1016/j.virusres.2009.07.014 PMID:19635508
20. Soysal $P$, Stubbs $B$, Lucato $P$, Luchini $C$, Solmi $M$, Peluso R, Sergi G, Isik AT, Manzato E, Maggi S, Maggio M, Prina AM, Cosco TD, et al. Inflammation and frailty in the elderly: A systematic review and meta-analysis. Ageing Res Rev. 2016; 31:1-8.

https://doi.org/10.1016/j.arr.2016.08.006

PMID:27592340

21. Forster $\mathrm{P}$, Forster L, Renfrew C, Forster $\mathrm{M}$. Phylogenetic network analysis of SARS-CoV-2 genomes. Proc Natl Acad Sci U S A. 2020; 117:9241-3. https://doi.org/10.1073/pnas.2004999117 PMID:32269081

22. Wang $Y$, Li J, Xia Y, Gong R, Wang K, Yan Z, Wan X, Liu G, Wu D, Shi L, Lau W, Wu M, Shen F. Prognostic nomogram for intrahepatic cholangiocarcinoma after partial hepatectomy. J Clin Oncol. 2013; 31:1188-95. https://doi.org/10.1200/JCO.2012.41.5984 PMID:23358969 


\section{SUPPLEMENTARY MATERIALS}

\section{Related computerized programs for nomogram with $\mathbf{R}$}

library (rms)

library (Hmisc)

library (grid)

library (lattice)

library (Formula)

library (ggplot2)

library (foreign)

library (pROC)

library (ggDCA)

library (verification)

\section{For nomogram}

rawdata $<-$ read.csv("nomo.csv")

$\mathrm{Y}<-$ rawdata $\$ \mathrm{Y}$

Age $<-$ rawdata $\$$ Age

Dyspnea $<-$ rawdata $\$ D y s p n e a$

LMPH $<-$ rawdata\$LMPH

IL6 $<-$ rawdata\$IL6

$\mathrm{CRP}<-$ rawdata\$CRP

$\mathrm{dev}=$ rawdata[rawdata $\$$ category $==0$, ]

$\operatorname{vad}=\operatorname{rawdata}[$ rawdata $\$$ category $==1$,

ddist $<-$ datadist(rawdata)

options (datadist $=$ 'ddist')

modelC2 $<-\operatorname{lrm}(\mathrm{Y} \sim$ Age + Dyspnea + CRP + IL6 + LMPH, data $=$ rawdata, $\mathrm{x}=$ TRUE, $\mathrm{y}=$ TRUE $)$

nom <- nomogram(modelC2, fun= function $(x) 1 /(1+\exp (-x))$, fun.at $=\mathrm{c}(0.01,0.03,0.1,0.25,0.5,0.7,0.9,0.99), \mathrm{lp}=\mathrm{F}$, funlabel = "Risk")

plot (nom)

\section{For resampling validation of nomogram}

Validate $($ modelC2, method $=$ "boot", $B=1000, d x y=T)$

\section{For computing the C-Index}

$\mathrm{r}<-\operatorname{rcorrcens}(\operatorname{Surv}(\mathrm{Y}) \sim \operatorname{predict}(\operatorname{modelC} 2)$, data $=$ rawdata $)$

$\mathrm{r}$

\section{For calibration curve}

cal $<-$ calibrate $($ modelC2, method $=$ 'boot', B=1000)

plot $(\mathrm{cal}, \mathrm{xlim}=\mathrm{c}(0,1.0), \mathrm{ylim}=\mathrm{c}(0,1.0))$

\section{For ROC curve}

modelC2 $<-\operatorname{lrm}(\mathrm{Y} \sim$ Age + Dyspnea + CRP + IL6 + LMPH, data $=$ rawdata, $\mathrm{x}=$ TRUE, $y=$ TRUE $)$

predict $<-\operatorname{predict}(\operatorname{modelC} 2)$

write.csv(predict, "predict1.csv")

rawdata $<-$ read.csv("cal000.csv")

$\mathrm{Y}<-$ rawdata $\$ \mathrm{Y}$

predict $<-$ rawdata\$predict

roc $($ rawdata $\$$, rawdata $\$$ predict, plot $=$ TRUE, print.thres $=$ TRUE, print.auc $=$ TRUE $)$ 
For DCA curve

$\mathrm{d}<-$ dca (modelC2)

ggplot (d) 